\title{
Transtorno obsessivo-compulsivo resistente: conceito e estratégias de tratamento
}

\author{
Roseli G Shavitta, Ygor A Ferrãob, M aria Claudia Bravoa, Cristina Belottoa, \\ Ademir Lopes J uniora e Eurípedes C M iguela
}

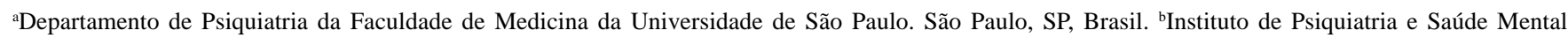
do Rio Grande do Sul. Rio Grande do Sul, RS, Brasil

\begin{abstract}
Despite the fact that majority of OCD patients responds to conventional treatments, about $40 \%$ remain symptomatic after appropriate trials with serotonin uptake inhibitors (SRIs) or behavioral therapy. A patient is considered treatment-resistant after failing to respond to three pharmacological trials with SRIs as well as to behavior therapy. When all somatic strategies have been tried, including augmentation with other medications, and the patient remains symptomatic, he or she is considered treatment refractory. The concepts of resistance and refractoriness are briefly revised, and the current augmentation strategies, as well as non-pharmacological approaches such as neurosurgery and transcranial magnetic stimulation, are addressed in this paper. Finally, issues of predictive response factors, pharmacogenetics, and the pathophysiology of resistant cases are suggested as targets for further researches.
\end{abstract}

Keywords

Obsessive-compulsive disorder. Treatment.

\section{Introdução}

O tratamento do transtorno obsessivo-compulsivo (TOC) encontra-se um pouco além do "meio do caminho" em termos de resultados alcançados. Felizmente, a maioria dos pacientes é beneficiada pelos tratamentos convencionais disponíveis. No entanto, ensaios clínicos com inibidores seletivos de recaptação da serotonina (ISRS) sugerem que até $40 \%$ dos pacientes não respondem satisfatoriamente ao tratamento com um desses agentes. ${ }^{1}$

Define-se como resistência ao tratamento a ausência de resposta aos tratamentos de primeira linha recomendados para o TOC, que são os inibidores de recaptação de serotonina (IRS) e a terapia comportamental (TC). ${ }^{2}$ É importante destacar que as definições de resposta satisfatória podem variar entre os estudos e, assim, influenciar os resultados.

O instrumento clínico mais amplamente utilizado para medir a intensidade dos sintomas é a escala Yale Brown de Sintomas Obsessivo-Compulsivos (Y-BOCS). ${ }^{1}$ Pode-se considerar como falta de resposta satisfatória um escore residual de 12 na YBOCS ou um escore final com redução menor que $25 \%$ em relação ao inicial. ${ }^{2}$

O termo refratário refere-se à situação de não-resposta a todos os tratamentos disponíveis, farmacológicos e psicoterápicos.

Neste artigo, faz-se referência aos pacientes resistentes e refratários ao tratamento, que constituem um grande desafio para os profissionais envolvidos na tarefa de ajudá-los.

\section{Avaliação da resistência ao tratamento}

O primeiro passo para constatar a resistência ao tratamento é uma avaliação cuidadosa do paciente para a revisão do diagnóstico principal $^{2}$ (veja artigo de Torres sobre diagnóstico diferencial de TOC neste volume). Confirmado o diagnóstico de TOC, passa-se a uma análise dos tratamentos já realizados: quais medicamentos foram usados, qual a dose máxima atingida para cada um, os motivos para não haver atingido as doses máximas (efeitos colaterais versus fatores limitantes ao aumento da dose que poderiam ter sido contornados), a duração de cada tratamento e os resultados obtidos em cada etapa. É importante também avaliar possíveis fatores familiares e ambientais que possam afetar negativamente a adesão ou os resultados terapêuticos que, em geral, exigem abordagem psicoterápica.

Por último, investiga-se a presença concomitante de outros transtornos psiquiátricos e clínicos que possam interferir na resposta ao tratamento e exigir uma terapêutica diferente daquela recomendada para o TOC isoladamente.

Uma vez analisados esses fatores, pode-se verificar a possibilidade de estar diante de um paciente com TOC resistente ou de recomeçar um tratamento convencional sob condições mais adequadas.

\section{Estratégia de tratamento dos casos resistentes}

Diante de um paciente com TOC resistente, pode-se recorrer a abordagens psicoterápicas e farmacológicas. A utilização da psicoterapia será abordada em outros artigos deste suplemento. Neste artigo, abordar-se-ão as estratégias farmacológicas, a eletroconvulsoterapia, a estimulação magnética transcraniana e a neurocirurgia.

Na Tabela 1, descrevem-se as orientações elaboradas a partir 
de um consenso entre especialistas, ${ }^{4}$ com alternativas de primeira, segunda e terceira linhas no manejo de pacientes com TOC resistente e refratário ao tratamento. Em seguida, a Tabela 2 descreve as alternativas farmacológicas estudadas até o momento, com comentários sobre cada uma delas. A Figura 1 mostra um roteiro seqüencial de tratamento proposto pelos autores, baseado na sua prática clínica.* Em suma, diante de pacientes resistentes aos IRS e à TC, após a utilização infrutífera da clomipramina, utilizam-se duas estratégias básicas: associamse ao IRS neurolépticos ou um ISRS com CMI. Se o paciente apresenta tiques ou ST ou traços esquizóides de personalidade, associam-se IRS e neurolépticos típicos como o pimozide. Casos resistentes em geral podem responder à associação de IRS e neurolépticos atípicos como a risperidona ou a olanzapina. Se a opção for pela associação de ISRS e CMI, geralmente inicia-se com o ISRS, e, em seguida, adicionam-se baixas doses de CMI (25 mg), sempre monitorando os níveis de CMI e a função cardíaca com ECG, aumentando a dose com muito cuidado para evitar convulsões e efeitos cardiovasculares adversos.

Comentar-se-ão, a seguir, os recursos farmacológicos não usuais, como a via endovenosa, e outros recursos não farmacológicos atualmente disponíveis para pacientes com TOC refratário ao tratamento.

\section{Administração parenteral de clomipramina}

Recentemente, Fallon et al ${ }^{22}$ compararam a administração de clomipramina $\mathrm{EV}$ com placebo $\mathrm{EV}$ em pacientes resistentes à clomipramina oral; seis (21\%) pacientes dos $29 \mathrm{com}$ clomipramina EV e nenhum do grupo-placebo mostraram resposta. ${ }^{22}$ Koran et al, comparando a administração de clomipramina EV em doses crescentes com pulsos de clomipramina EV em doses mais altas (150 mg a $200 \mathrm{mg}$ ), observaram um efeito mais rápido e marcante com a segunda estratégia ${ }^{23}$ Recomenda-se a monitorização cardíaca, da pressão arterial e do pulso durante as infusões. As infusões nos vários estudos costumam ser diárias, durante 14 dias, com doses variando, entre os estudos, de $75 \mathrm{mg} / \mathrm{dia}$ a $200 \mathrm{mg} / \mathrm{dia}$, podendose continuar o tratamento com a clomipramina por via oral. ${ }^{5,22,23}$

\section{Eletroconvulsoterapia}

A eletroconvulsoterapia pode ser interessante quando há comorbidade com depressão e, principalmente, risco de suicídio, não sendo indicada para sintomas obsessivo-compulsivos isolados dos sintomas depressivos., ${ }^{4}$

\section{Estimulação magnética transcraniana}

É um recurso ainda restrito ao âmbito da pesquisa, mas muito promissor para o uso clínico. Ela altera a atividade cerebral por meio de campos eletromagnéticos pulsáteis de alta intensidade que induzem correntes elétricas focais no córtex cerebral. Um estudo de Greenberg et $\mathrm{al}^{24}$ mostrou que a estimulação no córtex órbito-frontal direito em pacientes com TOC reduziu a premência, que durou oito horas, de realizar compulsões. Esse método parece alterar a atividade neuronal em áreas corticais envolvidas com o sentimento da necessidade de realizar compulsões.

\section{Estimulação cerebral profunda}

Gabriëls et al ${ }^{25}$ exploraram os efeitos da estimulação elétrica nas porções anteriores da cápsula interna de três pacientes com TOC refratário. Nesse local é colocado um eletrodo da espessura de um fio de cabelo que pode ser modulado. Ampararam o estudo na reversibilidade do procedimento, comparado à neurocirurgia, e por causar poucos efeitos adversos. Nos três pacientes, a Y-BOCS mostrou redução dos sintomas quando aplicada pelo psiquiatra; já na Y-BOCS autoaplicada, um paciente não teve melhora. Os avaliadores notaram alterações positivas na aparência, na atitude, na fala e no afeto. ${ }^{25}$ Esta é uma nova técnica que também pode ser promissora nos casos refratários, como uma alternativa menos invasiva que a neurocirurgia.

\section{Neurocirurgia}

A intervenção neurocirúrgica no TOC é indicada para pacientes adultos com quadro de duração superior a cinco anos - situação que causa sofrimento intenso ou prejuízo importante no funcionamento psicossocial -, para os quais todas as opções terapêuticas disponíveis utilizadas não tiveram efeito na redução dos sintomas ou tiveram de ser descontinuadas por efeitos adversos intoleráveis. ${ }^{26}$

Outro diagnóstico do Eixo I do DSM-IV (síndrome cerebral orgânico, transtorno delirante, transtornos pelo uso ou abuso de álcool ou drogas ilícitas) e os transtornos de personalidade do grupo A (paranóide) ou B (histriônico, limítrofe ou antisocial) contra-indicam a neurocirurgia, bem como a presença de complicações de Eixo III (atrofia ou tumores cerebrais). Já os transtornos de personalidade do grupo C (evitativo e obsessivocompulsivo) não são contra-indicações por poderem melhorar ou desaparecer após o procedimento. ${ }^{26}$

Os principais riscos da neurocirurgia são: infecção, hemor-

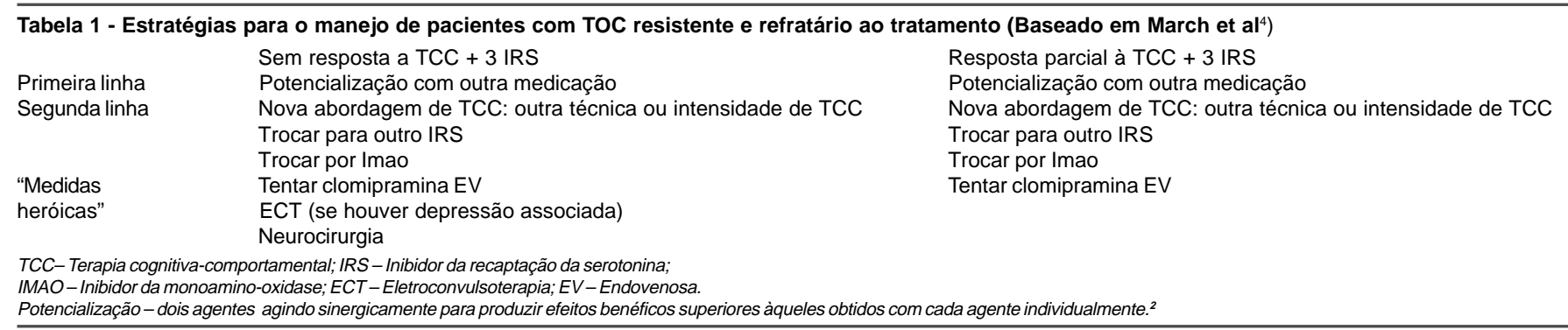

*Mercadante MT, Rosario-Campos MC, Shavitt RG, Brotto AS, Hounie AG, Souza MO, et al. Transtorno obsessivo-compulsivo. In: Louzã Neto MR, Motta T, Wang Y-P, Elkis H, editores. Psiquiatria Básica $2^{\text {a }}$ ed. (in press). 


\begin{tabular}{ll}
\hline Tabela 2 - Fármacos e substâncias que podem ser utilizadas em monot \\
Fármaco/substância & Mecanismo de ação \\
Lítio & Potencializa os neurônios serotoninérgicos \\
& pré-sinápticos em algumas regiões cerebrais \\
& e interfere na noradrenalina, na dopamina \\
& e no sistema de segundos mensageiros \\
& (proteínas $\mathrm{G})$. Dose varia de $300-1.500 \mathrm{mg} / \mathrm{dia}$. \\
& $\begin{array}{l}\text { Agonista serotoninérgico }\left(5-\mathrm{HT}_{1 \mathrm{~A}}\right) \text { pré e } \\
\text { pós-sináptico, além de agir em receptores } \mathrm{D}_{2} \text {. } \\
\text { Doses até } 60 \mathrm{mg} / \text { dia. }\end{array}$
\end{tabular}

\begin{tabular}{ll}
\hline Triptofano & Precursor da serotonina. Até $6 \mathrm{mg} / \mathrm{dia}$. \\
clonidina & Agonista alfa-2-adrenérgico.
\end{tabular}

Fenfluramina $\quad$ Propriedades de liberação e de recaptação serotoninérgica. Doses de 5-60 mg/dia. \begin{tabular}{ll}
\hline Naloxone e naltrexone & $\begin{array}{l}\text { Antagonistas opióides. Doses de } 0,3 \mathrm{mg} / \mathrm{Kg} \\
\text { de Naloxone EV e até } 300 \mathrm{mg} / \mathrm{dia} \text { de }\end{array}$
\end{tabular} Naltrexone VO.

Trazodone Antidepressivo atípico, inibidor da recaptação
da serotonina $\left(5 \mathrm{HT}_{1 \mathrm{~A}}, 5 \mathrm{HT}_{1 \mathrm{C}}\right.$ e $\left.5 \mathrm{HT}_{2}\right)$ e com efeito na adrenalina e dopamina.

\section{Outro IRSS + Parece reduzir a necessidade de doses}

Clomipramina elevadas de clomipramina, reduzindo intensidade e freqüência dos efeitos adversos. ${ }^{2,5}$ Acredita-se que a associação possa bloquear a recaptação da norepinefrina pelo efeito de seus metabólitos ou ter efeitos de bloquear a dopamina. ${ }^{7}$ As doses são muito variáveis.

Benzodiazepínicos O clonazepam é um benzodiazepínico que possui algumas propriedades serotoninérgicas $\left(5 \mathrm{HT}_{1}\right.$ e $\left.5 \mathrm{HT}_{2}\right)$. Não deve exceder $5 \mathrm{mg} / \mathrm{dia}$ e deve ser realizada por um período mínimo de 4 semanas.

\begin{tabular}{ll}
\hline Pindolol & $\begin{array}{l}\text { Antagonista beta-adrenérgico e de receptores } \\
5 \mathrm{HT}_{1 \mathrm{~A}} \text {. A dose recomendada é de } 2,5 \mathrm{mg}, \\
\text { três vezes ao dia. }\end{array}$ \\
\hline Inositol & $\begin{array}{l}\text { Precursor de alguns segundos mensageiros } \\
\text { (fosfatidilinositol) e está envolvido na } \\
\text { osmorregulação celular. Doses } \\
\text { de } 6-18 \mathrm{~g} \text { por dia. }\end{array}$ \\
\hline Triiodotironina & Hormônio tiroidiano. \\
\hline Oxitocina & Hormônio hipofisário
\end{tabular}

Eficaz em estudos abertos como potencializador de ISRS. ${ }^{12}$

Estudos de caso. Uso intranasal parece ser melhor. ${ }^{6}$

\section{Evidências favoráveis}

Séries de casos. ${ }^{5,6}$ Reduz sintomas

depressivos concomitantes. ${ }^{6}$

Evidências ou aspectos desfavoráveis Estudos com métodos mais adequados não mostraram eficácia após a quarta semana de associação com a fluvoxamina. $^{7}$

Relatos de caso, estudos abertos e comparados com placebo. ${ }^{5}$ Eficaz quando há comorbidade com $\mathrm{T}$. Ansiedade generalizada. ${ }^{6}$ Estudos de casos. ${ }^{9}$

Estudos de casos. ${ }^{2}$ Pode beneficiar pacientes com comorbidade com $\mathrm{T}$. Tourette. $^{2}$

Estudos de $\operatorname{casos}^{2}$ e aberto ${ }^{10}$ com bons resultados.

Pode ser útil em comportamentos de automutilação associados ao TOC e na tricotilomania. ${ }^{5}$

Estudos de casos. ${ }^{5,6}$ Indicado quando há insônia associada.

Estudos controlados com placebo. ${ }^{2,5,12}$

Estudos de caso e controlados. ${ }^{2}$ Indicação nas comorbidades com altos níveis de ansiedade, agitação psicomotora, transtorno afetivo bipolar ou insônia. Efeito anticonvulsivante (EEG alterado pode indicar o seu uso). ${ }^{6}$ Estudos controlados com placebo. ${ }^{14}$ Estudos comparados com placebo não mostram eficácia. ${ }^{8}$ Piora dos SOC em estudos de caso. ${ }^{2,6}$

Não existem estudos controlados. Sedação, resposta mínima em estudos abertos, não existem estudos controlados. $2,5,6$

Efeitos adversos: zunido, impotência e impulsividade sexual. ${ }^{2}$ Efeitos neurotóxicos. ${ }^{10}$

Resultados conflitantes quando usado para o TOC "puro". 5

Não funcionou em monoterapia quando controlado com placebo. ${ }^{11}$

Risco potencial de a clomipramina reduzir o limiar convulsivo. ${ }^{6}$ Pode alterar freqüência cardíaca e pressão arterial, além de alongar o intervalo QT no eletrocardiograma. ${ }^{6}$

Estudo controlado com placebo sem

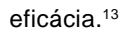

Parece incrementar respostas parciais e não tornar respondedores os não respondedores. ${ }^{12}$

Associado a IRS e controlado com placebo, não se mostrou eficaz. ${ }^{15}$

Estudo controlado não confirmou eficácia. $^{12}$

Efeitos adversos: distúrbios de memória, psicose e anormalidades osmóticas. ${ }^{6}$ Não existem estudos controlados para potencialização.

Antiandrogênicos

Sumatriptano

Psicoestimulantes

Morfina VO e Tramadol Ciproterona, espironolactona associada a testalactona, aminoglutetimide e flutamida. Agonista 5- $\mathrm{HT}_{1 \mathrm{D}}$ Dextro-anfetaminas ou metilfenidato. Doses de até $30 \mathrm{mg} / \mathrm{dia}$.

Opióides. Dose média de 37,5 mg /dia (+/- 8 $\mathrm{mg}$ ) para a morfina e $50-400 \mathrm{mg} /$ dia para o tramadol.

Anticonvulsivantes

Difenilhidantoín, ${ }^{5}$

Carbamazepina, ${ }^{5,6}$ ácido

valpróico, ${ }^{5,6}$ gabapentina. ${ }^{19}$

Doses variáveis.

Antipsicóticos

Antagonistas dopaminérgicos e com efeitos serotoninérgicos. Haloperidol, risperidona, pimozide, clozapina e olanzapina. Doses variáveis. A potencialização com neurolépticos deve ocorrer por no mínimo 4 semanas.

Imao Inibem a monoaminooxidase (MAO), enzima responsável pela formação da serotonina e outros neuroreceptores.
Relatos de casos. ${ }^{6}$

Relatos de casos. ${ }^{5}$

Série de casos. ${ }^{6}$ Não existem estudos controlados para potencialização. Série de casos mostrou piora dos SOC. ${ }^{16}$ Não existem estudos controlados para potencialização, nem evidências de sua eficácia em longo prazo.

Estudo de potencialização de IRS com morfina v.o.comparado com placebo mostrou efeito benéfico agudo. ${ }^{17}$ Estudo aberto com tramadol mostrou benefício como monoterapia. ${ }^{18}$ Estudos abertos e controlados com placebo. ${ }^{5}$ Indicados quando há relação dos sintomas obsessivo-compulsivos com epilepsia ou presença de alterações eletroencefalográficas típicas. ${ }^{5,19}$

Estudos duplo-cegos controlados com placebo na potencialização de IRS com risperidona ${ }^{20}$ e olanzapina. ${ }^{21}$ Eficazes quando há comorbidade com $\mathrm{T}$. de Tourette (haloperidol e pimozide) ${ }^{12} \mathrm{e}$ possivelmente no T. de personalidade esquizóide (olanzapina, risperidona) ${ }^{12}$ Eficaz em pacientes com T de pânico e fobia social concomitantes. ${ }^{2}$
Não há evidências de eficácia em longo prazo.

Ainda não existem dados conclusivos para o TOC na ausência de anormalidades no EEG.

Relatos de que a clozapina possa exacerbar os SOC. ${ }^{12}$ Riscos mais comuns da potencialização com neurolépticos: discinesia tardia, efeitos anticolinérgicos, acatisia e alteração eletrocardiográfica. ${ }^{5,6}$

Falta de estudos com metodologia adequada. A associação entre Imao e IRS ou Imao e buspirona pode levar a crises hipertensivas importantes ou a uma síndrome serotoninérigica grave, devendo-se, pois, evitar essa coadministração. ${ }^{2}$ 


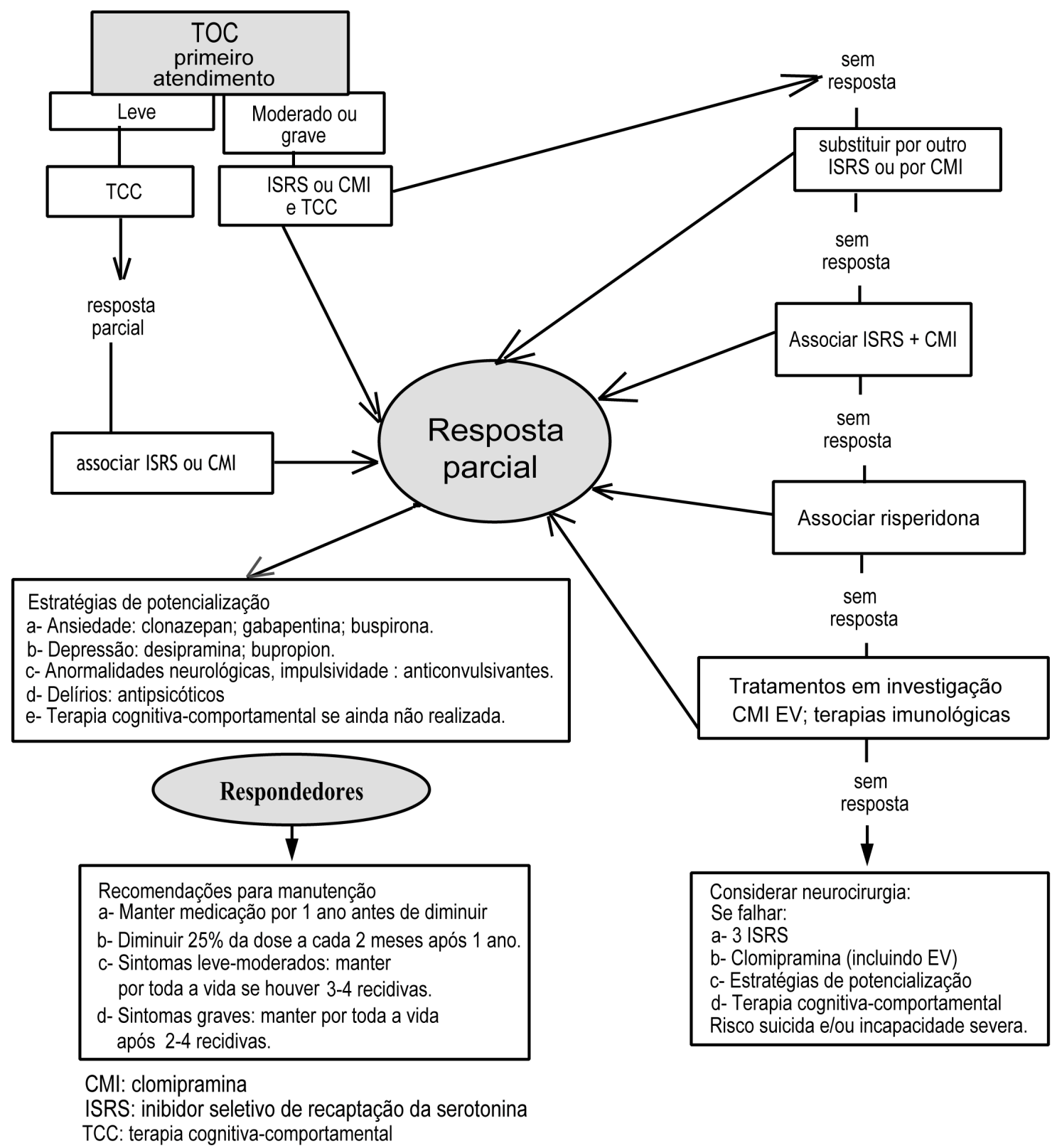

Figura - Roteiro de tratamento de pacientes com TOC segundo proposta dos autores. ${ }^{22}$

ragias, hemiplegia, convulsões, ganho de peso e alterações de personalidade. ${ }^{26}$ As funções cognitivas podem melhorar no pós-operatório. ${ }^{26}$

As técnicas mais utilizadas atualmente são:

1. tratotomia subcaudal: o alvo está na cabeça do núcleo caudado, numa região cerebral chamada de substância inonimada. Tem mostrado resultados benéficos em até $50 \%$ dos pacientes, embora faltem informações de seguimento em longo prazo; ${ }^{26}$

2. leucotomia límbica: efetua lesões dos quadrantes anteroinferiores dos lobos frontais e no feixe do cíngulo. Cerca de $80 \%$ dos pacientes submetidos a esse procedimento apresentaram melhora, que se manteve por mais de um ano; ${ }^{26}$
3. cingulotomia: as lesões ocorrem bilateralmente no cíngulo. Os índices de resposta ficam em torno de $30 \%$, e uma segunda intervenção pode ser necessária após 6 a 12 meses para ampliar a lesão inicial e melhorar o resultado terapêutico; ${ }^{26}$

4. capsulotomia: pode ser realizada com termolesão por radiofrequiência ou por raios gama. A vantagem dessa técnica é ser menos agressiva ao indivíduo, não sendo necessário abrir o crânio. $\mathrm{O}$ alvo é a perna anterior da cápsula interna. Rasmussen ${ }^{27}$ observou melhora em até $65 \%$ dos pacientes em avaliações feitas 6 e 12 meses após duas aplicações bilaterais de raios gama na porção anterior da cápsula interna. Mesmo restringindo o critério de melhora, $46 \%$ da amostra tiveram boa resposta ao procedimento. 
Todas essas intervenções são estereotáxicas e empregam recursos tecnológicos de alta precisão para ocasionar lesões bilaterais que podem ser determinadas por neuroimagem. Podem ser feitas com anestesia local e sedação leve. ${ }^{26}$

\section{Perspectivas para o futuro}

O estado atual do conhecimento sobre o tratamento do TOC permanece limitado. Um dos campos de pesquisa que poderiam contribuir para avançar nessa área diz respeito aos fatores preditivos de resposta ao tratamento. Por exemplo, se à heterogeneidade clínica do TOC corresponder uma heterogeneidade fisiopatológica, características clínicas específicas poderiam indicar tratamentos farmacológicos diferenciados para cada caso. Idealmente, os estudos deveriam ser pareados (head to head) e determinar a eficácia de diferentes estratégias de intervenção em amostras de pacientes bem caracterizados fenomenologicamente. ${ }^{28}$ Paralelamente, estudos de farmacogenética poderiam rastrear genes associados à resposta terapêutica a medicamentos específicos, a exemplo do que vem sendo feito na depressão. ${ }^{29}$

Quanto ao tratamento comportamental, estudos sobre fatores preditivos de resposta são recentes e se têm mostrado inconsistentes e de difícil reprodução. Apesar de sua eficiência, muitos pacientes não aderem às técnicas de exposição e prevenção de resposta ou desistem durante o tratamento. ${ }^{5} \mathrm{O}$ desenvolvimento de pesquisas nessa área será importante para indicações terapêuticas mais precisas. Com isto, métodos alternativos de tratamento poderiam surgir, melhorando os resultados. Aspectos ainda pouco investigados no impacto ao tratamento do TOC são: estrutura e funcionamento familiar; possí- veis subgrupos de TOC segundo a fenomenologia; temperamento e caráter como complementos ao estudo de personalidade; curso do TOC.

Entretanto, o desenvolvimento de técnicas de estimulação elétrica profunda ou por campos magnéticos, como na estimulação magnética transcraniana, pode não só trazer alívio para casos refratários mas também a possibilidade de maior compreensão do papel de várias estruturas cerebrais na etiologia do TOC.

\section{Conclusão}

A maior parte dos pacientes com TOC beneficia-se, pelo menos parcialmente, dos tratamentos de primeira linha disponíveis atualmente. Pacientes resistentes ao tratamento são aqueles que não respondem a vários desses tratamentos, enquanto os pacientes refratários são aqueles que não respondem a nenhum esquema terapêutico de primeira, segunda ou terceira linha disponíveis. Existem várias estratégias farmacológicas e psicoterápicas para o manejo desses pacientes, além de alternativas como a neurocirurgia e a estimulação magnética transcraniana. Atualmente são poucos os centros no mundo que oferecem essas abordagens, mas, embora ainda não sejam procedimentos correntes no Brasil, é desejável que futuramente constituam alternativas concretas para pacientes refratários ao tratamento.

Fonte de financiamento: Este trabalho foi financiado pela Fundação de Amparo à Pesquisa do Estado de São Paulo (Processos números 99/ 01548-0; 99/12205-7 para Schavitt RG e 99/08560-6 Miguel EC) e do Conselho Nacional de Desenvolvimento Científico e Tecnológico (Processo no 521369/96-7 para Miguel EC).

\section{Referências}

1. McDougle CJ. Update on pharmacologic management of OCD: agents and augmentation. J Clin Psychiatry 1997;58:11-7.

2. Rauch SL, Jenike MA. Management of treatment resistant obsessive compulsive disorder: concepts and strategies. In: textbook from the proceedings of the 1st IOCDC; 1993 March 12-13; Capri, Italy.

3. Goodman WK, Price LH, Rasmussen S, Mazure C, Fleischman RL, Hill CL, et al. The Yale-Brown obsessive-compulsive scale (YBOCS): Part I. Development, use and reliability. Arch Gen Psychiatry 1989;46:1006-11.

4. March JS, Frances A, Carpenter D, Kahn DA. The expert consensus guideline series: Treatment of obsessive-compulsive disorder. J Clin Pyschiatry 1997;58(4):1-72.

5. Jenike MA. Drug treatment of obsessive-compulsive disorders. In: Jenike MA, Baer L, Minichiello WE, editors. Obsessivecompulsive disorders: practical management. $3^{\text {rd }}$ ed. Mosby; 1998. p. $469-532$.

6. Goodman WK, McDougle CJ, Barr LC, Aronson SC, Price LH. Biological approaches to treatment-resistant Obsessive Compulsive Disorder. J Clin Psychiatry 1993;54:16-26.

7. McDougle CJ, Price LH, Goodman WK, Charney DS, Heninger GR. A controlled trial of lithium augmentation in fluvoxaminerefractory obsessive-compulsive disorder: lack of efficacy. J Clin Psychopharmacol 1991;11:175-84.
8. McDougle CJ, Goodman WK, Leckman JF, Holzer JC, Barr LC, McCance-Katz E, et al. Limited therapeutic effect of addition of buspirone in fluvoxamine-refractory obsessive-compulsive disorder. Am J Psychiatry 1993;150:647-9.

9. Rasmussen SA. Lithium and tryptophan augmentation in clomipramine resistant obsessive-compulsive disorder. Am J Psychiatry 1984;141:1283-5.

10. Hollander E, DeCaria CM, Schneider FR. Fenfluramine augmentation of serotonin reuptake blockade antiobsessional treatment. J Clin Psychiatry 1990;51:119-23.

11. Pigott TA, L'Hereux F, Rubenstein C, Berstein S. A double-blind, placebo controlled study of trazodone in patients with obsessivecompulsive disorder. J Clin Psychiatry 1992;12:156-62.

12. Zohar J, Sasson Y, Amitai K, Amiaz L, Sverdlic A, Chopra M. Pharmacological options for refractory OCD. In: Textbook of the $5^{\text {th }}$ International Obsessive-Compulsive Disorder Conference; 2001 March 29- April 1, Sardinia, Italy.

13. Pigott TA, L`Heureux F, Rubenstein CS et al. A controlled trial of clonazepam augmentation in OCD patients treated with clomipramine or fluoxetine. New Research Abstracts. APA Annual Meeting; 1992.

14. Dannon PN, Sasson Y, Hirschmann S, Iancu I, Grunhaus LJ, Zohar J. Pindolol augmentation in treatment-resistant obsessivecompulsive disorder: a double-blind placebo controlled trial. Eur Neuropsychopharmacol 2000;10:165-9. 
15. Fux M, Benjamin J, Belmaker RH. Inositol versus placebo augmentation of serotonin reuptake inhibitors in the treatment of obsessive-compulsive disorder: a double-blind cross-over study. Inter J Neuropsychopharmacol 1999;2:193-5.

16. Koran LM, Pallanti S, Quercioli L. Sumatriptan, 5-HT1D receptors and obsessive-compulsive disorders. In: Texbook of the 5 th International Obsessive-Compulsive Disorder Conference; 2001 March 29-April 1, Sardinia, Italy.

17. Franz B, Bullock KD, Elliot MA, Koran LM. Oral morphine in treatment resistant obsessive-compulsive disorder. In: Textbook of the 5 th International Obsessive-Compulsive Disorder Conference; 2001 March 29-April 1, Sardinia, Italy.

18. Shapira NA, Keck Jr, PE, Goldsmith TD, McConville BJ, Haggard PJ, McElroy SL. Open-label pilot study of tramadol hydrochloride in treatment-refractory obsessive-compulsive disorder. Depress. Anxiety 1997;6:170-3.

19. Greenberg BD, Corá-Locatelli G, Smith MJ, Nguyen MB, Keel J, Justement L, et al. Controlled study of gabapentin augmentation of fluoxetine in OCD: effects on symptoms and on cortical excitability. In: Textbook of the 5 th International ObsessiveCompulsive Disorder Conference; 2001 March 29-April 1, Sardinia, Italy.

20. McDougle CJ, Epperson CN, Pelton GH, Wasylink S, Price LH. A double-blind, placebo-controlled study of risperidone addition in serotonin reuptake inhibitor-refractory obsessive-compulsive disorder. Arch Gen Psychiatry 2000;57:794-801.

21. Bystritsky A, Ackerman DL, Rosen RM, Vapnik T, Gorbis E, Maidment KM, et al. Augmentation of SSRI response in refractory OCD using adjunctive olanzapine: a placebo-controlled trial. In: Textbook of the 5th International Obsessive-Compulsive Disorder Conference; 2001 March 29-April 1, Sardinia, Italy.
22. Fallon BA, Liebowitz MR, Campeas R, Schneier FR, Marshall R, Davies $\mathrm{S}$, et al. Intravenous clomipramine for obsessivecompulsive disorder refractory to oral clomipramine. Arch Gen Psychiatry 1998;55:918-24.

23. Koran LM, Pallanti S, Paiva RS, Quercioli L. Pulse loading versus gradual dosing of intravenous clomipramine in obsessivecompulsive disorder. Eur Neuropsychopharmacol 1998;8:121-6.

24. Greenberg BD, George MS, Martin JD, Benjamin J, Schalaepfer TE, Altemus M. Effect of pre-frontal repetitive transcranial magnetic stimulation in OCD: a preliminary study. Am J Psychiatry 1997;154:867-9.

25. Gabriëls L, Cosyns P, Nuttin B. Therapeutic effects of deep brain stimulation in patients with obsessive-compulsive disorder. In: Textbook of the 5 th International Obsessive-Compulsive Disorder Conference; 2001 March 29-April 1, Sardinia, Italy.

26. Jenike MA, Rauch SL, Baer L, Rasmussen SA. Neurosurgical treatment of obsessive-compulsive disorder. In: Jenike MA, Baer L, Minichiello WE, editors. Obsessive-compulsive disorders: practical management. $3^{\text {rd }}$ ed. Mosby; 1998. p. 592-610.

27. Rasmussen S. Anterior gamma capsulotomy for intractable OCD. In: Textbook of the 5th International Obsesive-Compulsive Disorder Conference; 2001 March 29- April 1, Sardinia, Italy.

28. Rauch SL, Jenike MA. Treatment resistant obsessive compulsive disorder: practical strategies for management. In: Textbook from the Proceedings of the 1st International Obsessive Compulsive Disorder Conference, Capri, Italy, March 12-13, 1993.

29. Smeraldi E, Zanardi R, Benedetti F, Di Bella D, Perez J, Catalano M. Polymorphism within the promoter gene and antidepressant efficacy of fluvoxamine. Molecular Psychiatry 1998;3:475-6.

Correspondência: Roseli G Shavitt

Instituto de Psiquiatria da FM/USP

R. Dr. Ovídio Pires de Campos, s/nº - 05403-010 São Paulo, SP, Brasil

E-mail: shavitt@mtecnetsp.com.br 